\title{
Konzepte für eine vorteilhafte Lehre unter Pandemiebedingungen
}

\author{
K. Eckert ${ }^{1,2}$, S. Heitkam ${ }^{1,2}$ \\ ${ }^{1}$ Professur für Transportprozesse an Grenzflächen, Institut für Verfahrenstechnik und Umwelttechnik, Fakultät Maschinenwesen, \\ TU Dresden \\ ${ }^{2}$ Institut für Fluiddynamik, Helmholtz-Zentrum Dresden-Rossendorf
}

\section{Abstract}

In diesem Report wollen wir aufzeigen, wie Lehre unter Pandemiebedingungen nicht nur bewältigt wurde, sondern tatsächlich zu einem Mehrwert für die Studierenden geführt hat. Dazu analysieren und vergleichen wir unsere Erfahrungen aus drei Lehrveranstaltungen. Alle Veranstaltungen wurden vollständig virtuell und live gehalten und abschlieBend von den Studierenden evaluiert. Das positive Feedback wird Einfluss in die zukünftige Gestaltung dieser Veranstaltungen auch nach Bewältigung der Pandemie haben.

In this report, we want to show how teaching under pandemic conditions was not only managed, but actually led to added value for the students. To this end, we analyse and compare our experiences from three courses. All courses were held completely virtually and live and were finally evaluated by the students. The positive feedback will have an influence on the future design of these events, even after the pandemic has been overcome.

*Corresponding author: kerstin.eckert@tu-dresden.de
\end{abstract}




\section{Einleitung}

Im SoSe2020 und WiSe2020/21 wurden durch unseren Lehrstuhl im Rahmen des wahlobligatorischen Moduls VNT-36 (K. Eckert) die Vorlesungen Fest-Fluid-Stoffaustauschprozesse (FFSAP) und Produkt- und Produktionsintegrierter Umweltschutz (PIUS) gehalten. Außerdem wurde die fakultative Vorlesung "Bubbles, Foam and Froth" (BFF, S. Heitkam) erstmals angeboten.

In FF-SAP legen wir die physikalisch-technischen Grundlagen für relevante Prozesse der Ressourcengewinnung (u.a. elektro- und hydrometallurgische Prozesse, Nitrifikation in Belebtschlammbecken, Festbettadsorption oder Partikelseparation) und des Recyclings (z.B. De-Inking-Flotation für Altpapier). Die PIUSVorlesung, die bis letztes Jahr von Dr. Brummack gehalten wurde, haben wir komplett neu aufgesetzt, um sie besser mit FF-SAP zu verlinken. PIUS widmet sich neben Umweltschutzstrategien wichtigen Aspekten der Kreislaufwirtschaft wie Ressourcenschonung und Nachhaltigkeit; hinzu kommt die Öko-Bilanzierung. Das Hauptziel unserer PIUS-Vorlesung besteht darin, die Studierenden zu einem kritischen und ideologiefreien Durchdringen von Problemen an der Schnittstelle Umweltschutz und Gesellschaft/Wirtschaft zu befähigen, Grundlagen vermitteln und dabei die im Projekt verwendeten Methoden und Ergebnisse in die Lehre einbringen.

Die Vorlesung BFF war rein fakultativ. Sie entstand in Anbindung an die Emmy-Noether Nachwuchsgruppe zum Thema „Fluiddynamik von Schäumen“, die seit Mai 2020 am Institut verankert ist. Die Vorlesung soll Interesse für Schäume wecken, Grundlagen vermitteln und dabei die im Projekt verwendeten Methoden und Ergebnisse in die Lehre einbringen.

\section{Umsetzung}

Alle Vorlesungen wurden zu der im Vorlesungsplan angekündigten Zeit per GoToMeeting bzw. Zoom unter Nutzung von PowerPoint gehalten. Hinzu kam alle 2 Wochen eine Rechenübung, angegliedert vorrangig an FF-SAP. Die aufgezeichneten Vorlesungen haben wir danach auf die $\mathrm{ZIH}$-Cloud gelegt und die Vorle-
sung-PDFs vor der Vorlesung auf OPAL gestellt. Die Modulprüfungen, die nach aktueller PO beide mündlich abzunehmen waren, haben wir als Präsenzprüfung abgenommen.

Als "Stiftmenschen“, die Vorlesungen über Tablett-PC live entwickeln, haben uns die Digitalsemester anfänglich vor erhebliche Probleme gestellt, da wir das Gefühl hatten, die Studierenden "schlafen" uns vor dem PC ein, wenn wir diese Konzept beibehalten. Daher ist das ganze Semester, wie bei sicher allen, viel Zeit in die Digitalisierung der Vorlesungen geflossen. Prinzipiell hat die dafür erforderliche Überarbeitung und Präzisierung der Vorlesung FF-SAP jedoch gut getan. Mit Schrecken haben wir jedoch festgestellt, dass wir dadurch deutlich schneller sind als bei der bisherigen Präsenzlehre. Gegengesteuert haben wir mit vier Maßnahmen: (i) Sachverhalte länger erklärt und (ii) meistens 15-20 min Wiederholung der vorangegangen Vorlesung anbieten. (iii) Zusätzliche Rechenaufgaben vorsehen, die bei verfügbarer Zeit bearbeitet werden können und so einen flexiblen Puffer bilden. (iv) Besondere Mühe haben wir uns gegeben, Vorlesungsinhalte mit aktuellen Forschungsthemen unseres Lehrstuhls bzw. unserer Abteilung am HZDR zu verknüpfen. Das ist auf Anklang gestoßen und hat uns aktuell enorm viele BelegArbeiterInnen beschert.

Die Vorlesung BFF verfolgt verschiedene Ziele und adressiert verschiedene Hörerkreise: den Studierenden der Verfahrenstechnik und Naturstofftechnik soll die Vorlesung das sehr spezielle aber hoch relevante Thema "Schäume" näher zu bringen. Des Weiteren sollen Studierende aller Fachrichtungen für Schaum begeistert werden und so als zukünftige Hilfskräfte oder Mitarbeiter gewonnen werden. Mir ihr sollten hier neben den Studierenden der Verfahrenstechnik auch Promovierende in den Ingenieurwissenschaften angesprochen werden. Viele internationale Promovierende verspüren einen Mangel an englischsprachigen Lehrveranstaltungen an der TU Dresden, insbesondere im Bereich der Strömungsmechanik. Da Promovierende entsprechende Lehrveranstaltungen benötigen, um ihr Rigorosum abzulegen, sollte mit der fakultativen Vorlesung BFF eine englischsprachige Alternative geschaffen werden. 
Da die Vorlesung im Wintersemester 2020/21 pandemiebedingt sowieso virtuell stattfinden musste, entstand der Gedanke die Vorlesung auch Studierenden und Promovierenden an anderen Universitäten zugänglich zu machen. Daher wurde die Vorlesungsankündigung über fachspezifische Emailverteiler und in entsprechenden Fachforen publiziert. Da aktuell europaweit keine vergleichbare Vorlesung angeboten wird, fand das Angebot insbesondere bei Promovierenden in Schaum-verwandten Themen gutes Interesse. In mindestens drei Fällen wurden Promovierende explizit von ihrem PI aufgefordert teilzunehmen. Insgesamt nahmen anfangs ca. 30 Hörer teil. Davon waren laut einer Mentimeter-Umfrage [1] zu Beginn ca. $20 \%$ Studierende der TU Dresden, 30\% Promovierende der TU Dresden und anderer Dresdner Forschungsinstitute sowie 50\% externe Wissenschaftler und Wissenschaftlerinnen. Die Teilnehmerzahl reduzierte sich im Verlauf des Semesters auf ca. 20, wobei vor allem Studierende der TU Dresden verloren gingen. Hier könnte eventuell durch Laborpraktika in Präsenz entgegengewirkt werden.

Problematisch an einer öffentlichen Ankündigung war die Zugangskontrolle. Ohne Zugangskontrolle bestand die Gefahr eines Missbrauchs oder einer Störung der Veranstaltung. Daher wurde der Link zum Zoom-Meeting nur auf Email-Anfrage individuell verteilt. Dieses Verfahren hat sich bewährt, denn es kam zu keinerlei Störungen.

Problematisch war auch die Organisation des Kurses über OPAL. Zwar konnten externe Personen hier mittels Passwort die Vorlesungsfolien herunterladen, jedoch konnten sie sich nicht in den Kurs einschreiben und auch nicht in den Foren aktiv sein.

Der Inhalt der Vorlesung BFF basierte im Wesentlichen auf drei Säulen. Erstens wurden naturwissenschaftliche Zusammenhänge vermittelt. Zweitens wurden Rechenaufgaben diskutiert, um die erlernten Zusammenhänge zu verinnerlichen. Und drittens wurden Beispiele für die Anwendung des Erlernten in akademischer und industrieller Praxis erläutert.

Diese Säulen wurden für das virtuelle Format angepasst.

Säule 1: Schäume sind generell ein sehr interdisziplinäres Forschungsfeld. Sie vereinen phy- sikalische, chemische, mathematische und ingenieurwissenschaftliche Ansätze. Da 50\% der Hörer externe Wissenschaftler und Wissenschaftlerinnen waren, gab es auch hier eine gewisse Streuung der Ausbildung. Eine Mentimeter-Umfrage zu Beginn ergab einen Anteil von $70 \%$ aus den Ingenieurwissenschaften, $20 \%$ aus der Physik und $10 \%$ aus der Chemie.

Aufgrund dieser Verteilung wurden nur wenige Vorkenntnisse vorausgesetzt. Stattdessen wurden fachspezifische Grundlagen umfangreich eingeführt bzw. wiederholt. In einer Präsenzveranstaltung hätte man hier vermutlich mehr Grundlagen vorausgesetzt und dann basierend auf dem unmittelbaren Feedback der Studierenden weitere Erläuterungen eingefügt. Basierend auf dem Feedback der Studierenden (Abbildung 1) konnten alle Hörer dem Inhalt gut folgen.

Säule 2: Die naturwissenschaftlichen Grundlagen wurden in jeder Veranstaltung mit 1-2 Rechenübungen untermauert. Diese wurden im Mittelteil der Veranstaltung durchgeführt um eine Pause zu erzeugen und die Hörer zu aktivieren.

Verglichen mit Rechenübungen in Präsenz gab es dabei sehr wenig Rückfragen oder Diskussionen. Vermutlich war es den Studierenden unangenehm, Fehler oder Unwissenheit für alle sichtbar zu präsentieren. Stattdessen gab es lediglich einen Wettbewerb, wer zuerst das richtige Ergebnis in den Chat schrieb. Hier sollte in zukünftigen Veranstaltungen eine Möglichkeit für anonyme Fragen geschaffen werden, z.B. über Mentimeter.

Säule 3: Da die Vorlesung ursprünglich für Studierende der Verfahrenstechnik konzipiert war, lag ein Schwerpunkt auf der praktischen Anwendung der naturwissenschaftlichen Grundlagen. Dazu wurden in jeder Veranstaltung themenbezogene aktuelle Messtechniken oder Simulationsmethoden diskutiert. An geeigneten Stellen wurde auch auf Wissenslücken und die Verbindung zur eigenen Forschung hingewiesen. Aufgrund der Teilnahme externer Wissenschaftler führte insbesondere Säule 3 zu sehr interessanten Diskussionen im Anschluss an die Veranstaltungen. Diese Diskussionen waren eine große Bereicherung, 
denn sie demonstrierten allen Zuhörenden, wie komplex und vielfältig Schaumforschung ist. Falls in zukünftigen Veranstaltungen keine externen Gäste mehr teilnehmen können, sollen stattdessen Gastredner eingeladen oder virtuell eingebunden werden um diese Vielfältigkeit zu demonstrieren.

Wie bei der Vorlesung BBF eben skizziert, war es auch bei PIUS erforderlich, neue Wege zu gehen. Die gesamte PIUS-Vorlesung war ein intellektuelles Abenteuer, das mich (KE) das ganze Semester am Rotieren gehalten hat. Ich habe selten in einem Semerster, neben Fachbüchern so viele Bücher zu aktuellen Themen gelesen und in eine Vorlesung eingebaut. Ein wirklich empfehlenswertes Buch, das ich mir erlaube zu zitieren, ist das von Christian Berg [2].

Da es im Unterschied zu FF-SAP bei PIUS kein vorgefertigtes Gerüst gab, an das man sich halten konnte, wurde nach folgenden Konzept verfahren.

(i) Wichtig war es, die Studierenden in der "Corona-Isolierung" zu aktivieren. Das wurde durch Einbeziehung aktueller, auch politischer Themen und $a b$ und an provokanter Thesen versucht. Großer Wert wurde darauf gelegt, die Studierenden aktiv zu einer Meinungsbildung anzutörnen. Nach der Vorlesung gab es in GoToMeeting immer noch eine ca. 10-15 min Nach-Session, wo mit interessierten Studierenden gemeinsam Probleme diskutiert wurden.

(ii) Nach dem Motto „warum sollen nur wir uns mit den Mühen der Digitalisierung herumschlagen" gab es im ersten Drittel der Vorlesungsreihe ein Podcast-Projekt. Hier wurden die Studierenden angeregt, sich wichtigen, vorlesungsrelevanten Problemen, z.B. Wirtschaftswachstum vs. Nachhaltigkeit, von mehreren Seiten kritisch zu nähern und zu einer Meinungsbildung zu kommen. Das wurde von 2/3 der Studierenden aktiv angenommen und es wurden in 2er, 3er und 4er Gruppen sehr schöne Beiträge mittels Audacity produziert. Diese wurden in den Upload-Ordner des OPAL-Kurses hochgeladen und waren so auch den anderen zugänglich. In einer lockeren Abendsitzung, die von den Studierenden selber angeregt wurde, sind wir die Podcasts nochmal durchgegangen. Die Podcasts wurden insgesamt als gewinnbringendes Unternehmen von allen angesehen: Die Studierenden fanden die Auseinandersetzung mit den Themen spannend und hatten Spaß in der Produktion mit Audacity und wir konnten etwas gegen die Corona-Isolation tun. Wir hatten die Chance zu sehen, wie unsere Studierenden ticken und waren sehr angetan von der Tiefe der Auseinandersetzung.

(iii) Die PIUS-Vorlesung beinhaltete auch Diskussionen zu Nachhaltigkeitskonzepten (wie Effizienz/Suffizienz/Konsistenz etc.), was auch eine Auseinandersetzung mit dem individuellen Lebensstil einschloss. Fakultativ gab es das Angebot, alles mal zu durchforsten, um den individuellen CO2-Fussabdruck zu berechnen. Hier haben so gut wie alle freiwillig mitgemacht und ihre Ergebnisse auf OPAL hochgeladen.

(iv) Life cycle assessment, d.h. Ökobilanzierung spielt mittlerweile in vielen Projekten eine Rolle. Uns war es daher wichtig, eine Einführung in das Thema gekoppelt mit einer praktischen Softwareübung mit freier Software (OpenLCA) anzubieten. Dieses Projekt hatte einen Umfang von 4 Vorlesungen: Einführung, Installationshinweise, und drei Übungsblöcke. In den Übungsblöcken gab es von uns formulierte Fragestellungen, die die Studierenden selbständig untersuchen sollten. Ausgangspunkt war das offizielle OpenLCA Tutorial zur Mineralwasserflaschen-Bewertung (Vorteil: gut dokumentiert, allen zugänglich). Dieses haben wir schrittweise um komplexere Fragestellungen (z.B. unterschiedliche Transportwege, Mehrfachbefüllungen etc.) erweitert. Diese 3 Blöcke, die eine free-style Variante einschlossen (sprich eine Fragestellung, die sich die Studierenden selber ausdenken sollten), waren im Zeitfenster der letzten 3 Vorlesungen (Juni/Juli) zu bearbeiten. Während dieser Zeit war der GoToMeeting-Kanal geöffnet und die Studierenden konnten Probleme mit uns diskutieren. Die gefundenen Lösungen waren auf OPAL hochzuladen.

Die uns ursprünglich von der Prüfungskommission genehmigte Variante, die OpenLCALösungen zu 30\% in der Prüfungsleistung einfließen zu lassen, haben wir am Ende doch 
nicht genutzt. Zu ungleich waren die technischen und sozialen Voraussetzungen bei den Studierenden. Es haben jedoch fast alle bei dem Projekt mitgemacht. 90\% haben mindestens einen Block geschafft; $70 \%$ haben Lösungen zu allen drei Blöcken hochgeladen.

\section{Unser Fazit}

Trotz des hohen zeitlichen Aufwands haben wir die Semester als gewinnbringend angesehen. Sehr positiv überrascht waren wir von den mündlichen Prüfungsergebnissen. In FFSAP waren keinerlei Unterschiede zu den letzten Jahren festzustellen. Besonders begeistert waren wir von den PIUS-Prüfungen. Es war eine Freude, die hohe Zahl von klug und kritisch argumentierenden Studierenden zu erleben.

Folgende Dinge sehen wir bei VNT-36 als verbesserungswürdig an: (i) Übungen in GoToMeeting sind ein schwieriges Unterfangen. Bei Präsenzübungen trauen sich die Studierenden immer mehr zu fragen; bei GoToMeeting eher weniger. Dann spricht man ins Mikro, ohne Studierende zu sehen, was wenig Spaß machte. Daher haben wir die Übungen meistens als Vorrechenübung gehalten mit einigen Fragen live aus dem Auditorium oder per Chat. Hier sind wir an einem Austausch zur Optimierung sehr interessiert. (ii) Die PDFs mussten aus Zeitgründen immer ohne weitere Bearbeitung, d.h. ohne bewusst gesetzte Lücken, hochgeladen werden. Hier fehlt noch eine effiziente Lösung. (iii) In einigen Vorlesungen wurde Mentimeter zur Aktivierung und zum Testen des Wissens bei konkreten Fragestellungen eingesetzt. Das hätte hier noch öfter erfolgen sollen. Aber es fehlte zum einen die Zeit für die Vorbereitung; zum anderen war der Leistungsumfang der kostenfreien Version deutlich weniger komfortabel als früher. Als Alternative bietet sich z.B. umfrageonline.com an.

\section{Feedback von den Studierenden}

Nach Abschluss der letzten Veranstaltung erfolgte eine anonyme Evaluierung der Vorlesung BFF. Abbildung 1 zeigt die Resultate.

Es ist zu erkennen, dass alle Teilnehmer den
Inhalt der Vorlesung interessant fanden. Die meisten Teilnehmer fanden den Umfang angemessen und konnten der Vorlesung auch digital gut folgen. Die online durchgeführten Übungsaufgaben bewerteten alle Teilnehmer

\section{The content was interesting} strongly agree agree disagree strongly disagree

The amount of content was much too high too high adequate too low

The lecture was easy to follow strongly agree agree disagree strongly disagree

\section{Exercise supported understanding}

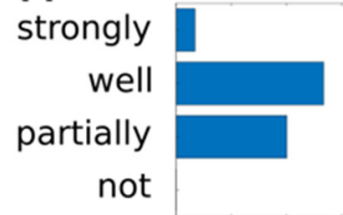

Additional time for processing no time

$<10 \mathrm{~min} /$ week $>10 \mathrm{~min} /$ week $>1 \mathrm{~h} /$ week

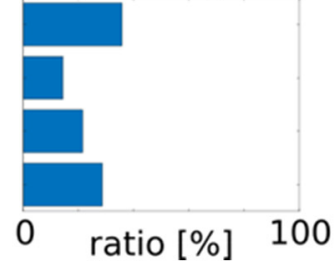

Abb. 1: Anonyme Evaluation der Vorlesung BFF im Wintersemester 20/21

als hilfreich. Diese Punkte sprechen für eine er folgreiche Umsetzung der virtuellen Veranstaltung mit dem gewählten Format. Allerdings verwendeten $50 \%$ der Teilnehmer weniger als 10 Minuten pro Woche, teilweise sogar überhaupt keine Zeit für die Vor- und Nachbereitung der Veranstaltung. Das könnte an der fakultativen Natur der Veranstaltung liegen, aber eventuell auch an einem Gefühl der Anonymität im virtuellen Raum. Schlechte Vorbereitung der Studierenden fällt nicht auf. 
Die Vorlesung FF-SAP war in der offiziellen Lehrveranstaltungsevaluation und wurde mit einer Gesamtnote von 1.27 bewertet, was uns sehr gefreut hat. Die verbliebenen Kritiken (ab und an bessere Strukturierung, mehr Prüfungsfragen in die Kapitel direkt einbauen, Quiz am Anfang) versuchen wir umzusetzen.

Ebenso motivierend war das spontane Feedback mehrerer Studierender nach der mündlichen PIUS-Prüfung. Sie hätten selten so viele spannende Diskussionen untereinander zu Themen einer Vorlesung geführt wie in diesem Semester.

Das bestärkt uns, wissenschaftliche Inhalte $a b$ und an verknüpft mit aktuellen und z.T. politischen Fragestellungen anzubieten, um dadurch die Studierenden zu aktiver Stellungnahme und Meinungsbildung anzuregen.

\section{Ausblick auf ein hybrides Lehrangebot}

Wir können uns vorstellen, einige der Vorlesungen auf 30 min Online-Video zu einem bestimmten Thema zu verkürzen. Die frei gewordene Zeit würden wir zum einen dafür einsetzen, dass die Studierenden über das Problem nachdenken müssen und Lösungen dafür finden sollen. Zum anderen für einen Konsultations- und Diskussionstermin, bei dem die aufgetretenen Fragen und gefundenen Lösungen diskutiert werden.

Im Bereich der Schäume existiert scheinbar Europaweit keine dezidierte Vorlesung. Aufgrund des sehr positiven Feedbacks der internationalen Studierenden und Promovierenden auf die Vorlesung BFF könnte die Vorlesung auch zukünftig virtuell und offen durchgeführt werden. Alternativ wäre auch ein livestream aus dem Hörsaal denkbar. Neben dem reinen Lehrauftrag würde das auch stark der internationalen Vernetzung und dem studentischen Austausch dienen. Jedoch soll für Studierende in Präsenz zukünftig ein Laborpraktikum in Präsenz angegliedert werden.

\section{Danksagung}

Ein herzlicher Dank geht zum einen an Dr. K. Schwarzenberger für die OPAL-Unterstützung der Vorlesung und für die Übernahme des Vorlesungsblocks zur Entschwefelung sowie an unseren Tutor, Robin Wolf, für die effektive Unterstützung der Übungen zur Ökobilanzierung. Zum anderen danken wir allen Studierenden im Recycling-Modul 2020 für ihr enormes Interesse und Engagement, das die Vorlesungen auch für mich zu einem inspirierenden Erlebnis hat werden lassen.

\section{Literatur}

[1] https://www.mentimeter.com

[2] Christian Berg, Ist Nachhaltigkeit utopisch? Der neue Bericht an den Club of Rome, oe-kom-Verlag (2020) 